\title{
Los retos en la gestión de la innovación para América Latina y el Caribe: un análisis de eficiencia
}

\author{
José G. Aguilar-Barceló y Fernanda Higuera-Cota
}

\section{Resumen}

Con datos del Índice Global de Innovación (IGI) de 2016 (Universidad de Cornell/ INSEAD/OMPI, 2016) y apoyo del análisis envolvente de datos (AED) se evalúa la eficiencia de las 19 economías de América Latina y el Caribe en la creación de entornos favorables para la innovación. El mejor desempeño en la región se consigue en materia de infraestructura y adaptación de las tecnologías de la información y las comunicaciones (TIC), pero se evidencian problemas en formación de capital humano, desarrollo e impacto de la investigación y aspectos institucionales. Países como Chile y Colombia mostraron un producto inferior al esperado de acuerdo con sus factores, por lo que sus buenos resultados en innovación no van a la par de la gestión de la eficiencia. Fortalecer el funcionamiento de los mercados (competencia, crédito e inversión) y las capacidades para la absorción del conocimiento es uno de los principales retos para la región.

\section{Palabras clave}

Innovaciones, evaluación, datos estadísticos, recursos humanos, cambio tecnológico, competitividad, metodología estadística, América Latina y el Caribe

\section{Clasificación JEL}

O32, D24, F63

\section{Autores}

José G. Aguilar-Barceló es profesor e investigador de tiempo completo en la Facultad de Economía y Relaciones Internacionales de la Universidad Autónoma de Baja California (México). Correo electrónico: gaba@uabc.edu.mx.

Fernanda Higuera-Cota es profesora en la Facultad de Contaduría y Administración de la Universidad Autónoma de Baja California (México). Correo electrónico: higuera. maria@uabc.edu.mx. 


\section{Introducción}

En el pasado se entendía que el desarrollo socioeconómico y el crecimiento de los países dependía principalmente de sus dotaciones de capital y de mano de obra, mientras que otros recursos menos tangibles, como el nivel de información y de formación, se consideraban factores externos que solo influían indirectamente en el comportamiento de los mercados. Con el paso del tiempo, no obstante, se ha ido madurando la idea de que la gestión del conocimiento es un determinante del nivel de productividad, tanto de las economías modernas como de las tradicionales (Cañibano, 2005). Esta nueva concepción global está exigiendo cada vez más empleos centrados en el desarrollo de ideas innovadoras que puedan aplicarse a productos, servicios y procesos.

El fomento a la innovación, entendida como la capacidad para generar, adquirir, adaptar y usar nuevos conocimientos a fin de lograr ventajas competitivas sostenibles y acumulativas en el tiempo (OCDE/Eurostat, 2005), es un elemento que se encuentra contemplado en los planes de desarrollo de todos los países del primer mundo donde se ha fortalecido la política de innovación como elemento central de la política industrial ${ }^{1}$. Aunque América Latina y el Caribe cuenta con una larga tradición en materia de políticas de desarrollo científico y tecnológico, y cada vez presenta una mayor inversión en innovación (Bárcena, 2008), no le está siendo sencillo revertir el déficit en esta área a través del fortalecimiento de su política de innovación ${ }^{2}$. Esto se debe, entre otros factores, a que los países que conforman la región enfrentan retos propios de las economías emergentes, tales como fallas de coordinación entre empresas, altos niveles de corrupción e informalidad, escasez de capital humano calificado y limitada capacidad de actuación del Gobierno (Navarro y Olivari, 2016), lo que reduce la eficacia en la implementación de políticas públicas.

De acuerdo con el Banco Interamericano de Desarrollo (BID), durante 2016 las economías de América Latina y el Caribe presentaron un marcado déficit respecto de la incorporación de conocimiento y tecnología a sus procesos productivos. De ahí la importancia del análisis y entendimiento de las fallas de mercado que hacen que la inversión en innovación sea insuficiente y se realice de forma espontánea e intermitente, lejos de los niveles socialmente deseables. Un reto añadido es la medición de la eficiencia de las políticas enfocadas a la generación de entornos innovadores y su cuantificación en términos de retornos económicos.

La Organización de Cooperación y Desarrollo Económicos (OCDE) señala que la estimación confiable de la capacidad de innovación es fundamental para el adecuado diseño de políticas públicas y destaca que las mediciones que se llevan a cabo en la actualidad no reflejan integralmente el papel que esta desempeña en la economía contemporánea (OCDE, 2012). Ante este panorama, la presente investigación se centra en el análisis del aprovechamiento de la dotación de insumos para la creación de entornos que favorezcan el desarrollo de los países de América Latina y el Caribe en materia de innovación, a través de la medición de los retornos. En el trabajo se presenta una visión alternativa acerca de la gestión de la innovación en el contexto de sus indicadores agregados más importantes por cuanto se vinculan los resultados individuales para crear referencias regionales. La hipótesis por contrastar es que en los países de América Latina y el Caribe no siempre se cumple que un mayor nivel de insumos de innovación denote una economía más sólida y con mayor potencial de desarrollo, lo que se traduce en mayores y mejores productos de innovación, con lo que se deja ir una invaluable oportunidad para acortar las distancias con los países desarrollados.

\footnotetext{
1 La política de innovación puede entenderse como la amalgama de la política industrial y la política de ciencia y tecnología (OCDE/Eurostat, 2005).

2 De acuerdo con Bárcena (2008), entre 1990 y 2003 el gasto público en investigación y desarrollo (I+D) creció aproximadamente un $40 \%$ en esta región.
} 
Aquí se busca medir la eficiencia en la gestión de la innovación a través del análisis estadístico de una serie de variables observables obtenidas del Índice Global de Innovación de 2016. Este índice permite conocer la clasificación anual de las principales economías del mundo en cuanto al desempeño de la innovación y su incidencia en el desarrollo. Las economías incluidas en el índice representan el 92,8\% de la población mundial y el 97,9\% del PIB mundial.

El artículo se organiza en seis secciones, incluida esta introducción. En la sección II se describe el marco teórico que conduce al bosquejo del panorama internacional en materia de innovación y eficiencia, y se presentan los principales retos de los países en desarrollo para crear y aprovechar entornos de innovación. En la sección III se explican las variables y su funcionamiento, mientras que en el apartado IV se describe la metodología empleada. En la sección $\vee$ se interpretan y discuten los resultados derivados del análisis estadístico. Por último, en la sección VI se recogen las principales conclusiones y líneas de trabajo futuro.

\section{Marco teórico}

\section{Clarificación de algunos conceptos básicos de innovación y eficiencia}

De acuerdo con la Comisión Europea (1995), la innovación obedece a la transformación de una idea en un producto, servicio o procedimiento de fabricación o distribución, ya sea nuevo o mejorado, mediante el uso del conocimiento. Resulta entonces evidente que la innovación es especialmente relevante para el desarrollo y la competitividad de las economías emergentes. La OCDE (2012) describe cómo es que la innovación puede marcar la diferencia al momento de abordar los retos que se les presentan a dichas economías, tales como la erradicación de enfermedades, el abatimiento de la pobreza y la inseguridad pública, o la transferencia y adaptación de tecnología para la modernización productiva.

En el último siglo se han presentado propuestas explicativas para alcanzar un mayor entendimiento de la innovación, especialmente en su concepción como proceso. Entre las principales se encuentra el modelo de empuje de la tecnología, que contempla el desarrollo del proceso de innovación, teniendo como origen la ciencia y tecnología, hasta llegar a la comercialización de un bien o proceso económicamente viable (Rothwell, 1994). El énfasis en el papel del mercado como fuente de ideas innovadoras y determinante del curso de la I+D llevó más adelante al diseño del modelo de tirón de la demanda. Para Saren (1984), que adopta otra perspectiva, el proceso de innovación, desde que una idea se convierte en insumo de innovación hasta que este insumo llega a ser producto, ocurre por etapas en términos del funcionamiento de los departamentos de una empresa.

Otra propuesta es el modelo de enlaces en cadena que tiene distintas vías, basadas en la información y el conocimiento, que conectan las tres áreas de relevancia en el proceso de innovación tecnológica - la investigación, el conocimiento y la cadena central del proceso de innovación tecnológica -, y cuyo éxito exige la interacción entre las capacidades tecnológicas y las necesidades del mercado (Kline y Rosenberg, 1986). Finalmente, el modelo de triple hélice de Etzkowitz y Leydesdorff (2000) es el más completo entre los descritos, ya que en él se considera de forma conjunta el papel de la empresa, el Estado-Gobierno y el sector académico ${ }^{3}$. La triple hélice se considera como un modelo espiral de innovación debido a que capta distintas relaciones en múltiples puntos del proceso de capitalización del conocimiento.

\footnotetext{
3 Recientemente se han empezado a implementar modelos de innovación de cuádruple hélice donde la sociedad se integra a los componentes de la triple hélice.
} 
De acuerdo con Restrepo y Villegas (2007), todo proceso productivo involucra la utilización de recursos para transformar entradas en salidas con el objetivo de satisfacer ciertas necesidades. De ello deriva una serie de términos utilizados en el debate sobre la gestión y administración de los recursos, que permiten establecer pautas para la formulación de políticas públicas y su implementación, tales como productividad, eficacia y eficiencia. La productividad puede definirse como la relación entre la cantidad de bienes producidos y de recursos utilizados (Carro y González, 2015). Se trata de una herramienta para evaluar el rendimiento de los factores que forman una sociedad, país, organización o individuo. La eficacia es la virtud de lograr los resultados esperados, sin que ello suponga la maximización de la capacidad productiva de los insumos. Contrariamente al concepto anterior, la eficiencia sí implica la maximización del beneficio o la minimización del costo, lo que se traduce en una mayor obtención de productos con el mínimo de insumos, o, dicho de otra forma, el aseguramiento de la correcta distribución de los medios empleados en relación con los fines obtenidos (Quindós, Rubiera y Vicente, 2003) ${ }^{4}$.

\section{Creación de entornos de innovación y valoración de su retorno económico}

Bajo este enfoque de recursos y capacidades, un sistema de innovación debe estar integrado por los distintos actores públicos y privados que reúnan las mejores capacidades e insumos técnicos, comerciales y financieros en el ánimo de propiciar un entorno favorable. El Gobierno siempre será uno de estos actores. Su papel como ejecutor de una política de innovación potencialmente eficiente va más allá del diseño de la normatividad en la materia y la vigilancia de su aplicación. También debe servir como facilitador en la articulación y puesta en marcha de las iniciativas innovadoras y asumir el compromiso de formar una población receptiva y creativa (Banco Mundial, 2010).

Para Guimón (2004), la I+D juega un papel clave en el desempeño de los diferentes sectores industriales nacionales, inclusive en los más tradicionales (como el sector agrícola). El autor destaca que no solo se debe apostar por la creación de nuevo conocimiento, sino que también es necesario crear entornos y desarrollar capacidades que permitan que este se pueda absorber. Es ahí donde el papel de los Gobiernos es fundamental.

La Organización Mundial de la Propiedad Intelectual (OMPI) (Universidad de Cornell/INSEAD/ OMPI, 2016) sostiene que el Gobierno puede intervenir de dos maneras para corregir el problema de la baja inversión privada en I+D: explícitamente, a través de inversiones en sectores que se consideren clave para el desarrollo económico, o implícitamente, mediante la estructuración de políticas que faciliten la creación de entornos favorables a la innovación. Asimismo, la OCDE (2012) destaca que la innovación no solo trata de la generación de productos de alta tecnología o del incremento de las capacidades de aprendizaje, sino que también se relaciona con la búsqueda de las vías para maximizar el aprovechamiento de tecnologías como las TIC, ya que estas son un elemento clave para poder difundir el conocimiento y franquear las barreras tecnológicas.

Dada la limitación de recursos, el proceso para determinar los elementos a considerar en la correcta valoración de los retornos económicos de la innovación no es único. Generalmente se opta por la utilización de indicadores de salida, también denominados "productos de la innovación", y en menor medida se eligen indicadores asociados a los factores que la propician. Para Atilano, Mercado y Casanova (2015), los productos de la investigación son los parámetros que establecen una línea base para la evaluación del proceso mismo, al ser comparados con los resultados previstos.

\footnotetext{
4 Restrepo y Villegas (2007) sostienen que los términos "productividad" y "eficiencia" se han usado tradicionalmente como sinónimos para medir el desempeño de los procesos productivos.
} 
Para la Fundación COTEC (2001), la innovación requiere inversiones en activos tangibles e intangibles que puedan incrementar el crecimiento económico a través de dos vías: un cambio en las estructuras productivas (referida al incremento del esfuerzo innovador y la recomposición del gasto tecnológico) y un efecto multiplicador intensivo en áreas específicas que puedan presentar un rezago tecnológico o representar una oportunidad estratégica. A partir de ello se puede inferir que existen múltiples indicadores que permiten acercarse a la medición de los retornos económicos de la innovación, cuya composición muestra diferencias de grado y clase ${ }^{5}$.

\section{Retos para los países en desarrollo en materia de innovación}

Bogliacino y otros (2009) señalan que la innovación es empujada por la industrialización e impulsada por el crecimiento de los mercados. Sin embargo, para que la innovación alcance su mayor impacto social es necesario que los esfuerzos de investigación estén dirigidos a encontrar soluciones a las problemáticas de la población más necesitada y a incrementar su bienestar, tarea que, sin duda, involucra tanto a entes privados como públicos (Banco Mundial, 2010).

La innovación implica procesos distintos según se trate de un país desarrollado o en desarrollo (Bogliacino y otros, 2009). Las estrategias de fomento a la innovación de las economías de altos ingresos suelen incluir la creación de condiciones que promuevan la movilidad de factores en los mercados, la apertura al comercio y la inversión ${ }^{6}$. Esto podría aplicarse a las economías en desarrollo siempre que los procedimientos se adapten a las condiciones y necesidades locales (Navarro y Olivari, 2016). No obstante, las economías emergentes deberían poner énfasis en la gestión del conocimiento como estrategia para acortar distancias con el mundo desarrollado.

En el caso de los países desarrollados se requiere de una fuerte capacidad de I+D y una infraestructura en ciencia y tecnología para adquirir y - sobre todo-desarrollar los conocimientos y las competencias necesarias para operar en la frontera tecnológica, mientras que para los países en desarrollo el cambio tecnológico se produce a través de la adquisición de maquinaria y la imitación de productos y procesos desarrollados previamente en las economías avanzadas.

\section{Análisis de variables e indicadores}

\section{Composición del Índice Global de Innovación}

El Índice Global de Innovación permite conocer la clasificación anual de 128 países en cuanto a su grado de innovación y el desempeño que tienen sus sistemas nacionales de innovación en el contexto económico mundial. El índice tiene por objeto captar las facetas multidimensionales de la innovación y proporcionar las herramientas que puedan ayudar a adaptar las políticas para promover el incremento de la productividad a través de la creación de un entorno en el que tanto los factores que propician la innovación como sus productos se encuentren en constante evaluación.

Este índice está compuesto de 21 indicadores agrupados en 7 pilares: 15 indicadores de entrada (aquellos que propician la innovación) y 6 indicadores de salida (aquellos que son producto

\footnotetext{
5 Desde la perspectiva del Índice de Competitividad Global, la medición de la innovación se relaciona con las capacidades tecnológicas de las empresas, la inversión pública en innovación, la calidad de las instituciones de investigación científica y la colaboración entre empresas y universidades (Schwab, 2016).

6 La movilidad de factores en los mercados se refiere a la capacidad de adaptación de los factores productivos, especialmente del factor trabajo.
} 
de la innovación) (véase el cuadro 117. A su vez, el Índice Global de Innovación presenta cuatro medidas básicas de innovación: i) el subíndice de entrada, entendido como el promedio de las puntuaciones de los cinco pilares de entrada; ii) el subíndice de salida, que resulta del promedio de las puntuaciones de los dos pilares de salida; iii) el índice general, que es el promedio de los subíndices de entrada y salida, y iv) la razón de eficiencia, que se obtiene del cociente entre el subíndice de salida y el subíndice de entrada.

Cuadro 1

Composición del Índice Global de Innovación (IGI), 2016

\begin{tabular}{lll}
\hline Clase de pilar & Pilar (identificador) & Indicadores del pilar \\
\hline \multirow{2}{*}{ Entrada } & Institucional (P1) & Ambiente político, entorno regulatorio y ambiente de negocios. \\
\cline { 2 - 3 } & $\begin{array}{l}\text { Capital humano e } \\
\text { investigación (P2) }\end{array}$ & Educación secundaria, educación terciaria e investigación y desarrollo (l+D). \\
\cline { 2 - 3 } & \begin{tabular}{l} 
Infraestructura (P3) \\
\cline { 2 - 3 }
\end{tabular} & $\begin{array}{l}\text { Tecnologías de la información y las comunicaciones (TIC), } \\
\text { infraestructura y sostenibilidad ecológica. }\end{array}$ \\
\cline { 2 - 3 } & Sofisticación del mercado (P4) & Crédito, inversión, competencia comercial y escala de mercado. \\
\hline \multirow{2}{*}{ Salida } & Conocimiento y tecnología (P6) & Creación de conocimiento, impacto del conocimiento y difusión del conocimiento. \\
\cline { 2 - 3 } & Creatividad (P7) & Activos intangibles, servicios de bienes creativos y creatividad digital.
\end{tabular}

Fuente: Elaboración propia, sobre la base de Universidad de Cornell/INSEAD/Organización Mundial de la Propiedad Intelectual (OMPI), The Global Innovation Index 2016: Winning with Global Innovation, Ginebra, 2016.

Desde su propia concepción parcializada, las primeras dos medidas (los subíndices de entrada y salida) hacen patentes sus limitaciones en la determinación de la eficiencia. Por su parte, el índice general toma en cuenta, en un mismo sentido (es decir, no confronta unos con otros), tanto los elementos de entrada como de salida, lo cual, aunque internaliza el nivel de los primeros, no abona a la determinación del aprovechamiento de estos en la obtención de los segundos, y, por lo tanto, de la eficiencia. Finalmente, la razón de eficiencia, si bien toma en cuenta el diferencial entre elementos de entrada y de salida (dando un carácter a los primeros como generadores de los segundos), no relativiza este diferencial en términos del comportamiento de un conjunto de países similares ${ }^{8}$.

Debido a la conveniencia de que la medición de la eficiencia se haga entre unidades semejantes, el presente estudio se limita espacialmente al conjunto de países de América Latina y el Caribe. Se asume entonces que los países de este grupo comparten las problemáticas económicas y sociales relacionadas con el desarrollo de la capacidad innovadora, más allá de que existan diferencias -en algunos casos muy significativas - en los niveles de ingreso y desarrollo humano, el tipo de régimen político o los patrones de innovación (Bogliacino y otros, 2009)9 . Esta región es considerada como de gran potencial inexplorado en materia de innovación, pero también de riesgos importantes y latentes en el corto plazo asociados con la desigualdad y la vulnerabilidad social.

Al echar un vistazo a los datos del Índice Global de Innovación de 2016 se encuentra que Chile estuvo a la cabeza de América Latina y el Caribe en el índice general y en el lugar 44 de la clasificación mundial, seguido de Costa Rica, tanto en la tabla regional como en la mundial ${ }^{10}$. Con el puesto 61 en la clasificación mundial, México alcanzó la tercera mejor posición regional. En el cuadro 2 se muestran los puntajes y las posiciones del índice general, así como las categorías de ingreso según el PIB per

\footnotetext{
7 Los 21 indicadores, a su vez, surgen de 81 variables asociadas a la innovación.

8 Es decir, no genera referencias que no surjan de las simples comparaciones transversales o temporales.

9 Al final de la sección IV se justifica este tratamiento de la información.

10 El primer lugar mundial fue para Suiza, seguido de Suecia y el Reino Unido.
} 
cápita para los países de América Latina y el Caribe. Se presentan también la razón de eficiencia y la clasificación de los países respecto de dicha razón.

\section{Cuadro 2}

América Latina y el Caribe: indicadores de innovación, ingreso y eficiencia de acuerdo con el Índice Global de Innovación (IGI), 2016

\begin{tabular}{|c|c|c|c|c|c|c|}
\hline País (abreviatura) & $\begin{array}{l}\text { Índice } \\
\text { general } \\
(1)\end{array}$ & $\begin{array}{l}\text { Clasificación por } \\
\text { índice general } \\
\text { (1) }\end{array}$ & $\begin{array}{c}\text { Categoría de } \\
\text { ingreso } \\
\text { (con base en el } \\
\text { PIB per cápita) } \\
\text { (2) }\end{array}$ & $\begin{array}{l}\text { Clasificación } \\
\text { por categoría de } \\
\text { ingreso } \\
\text { (2) }\end{array}$ & $\begin{array}{l}\text { Razón de } \\
\text { eficiencia } \\
\text { (3) }\end{array}$ & $\begin{array}{l}\text { Clasificación por } \\
\text { razón de eficiencia } \\
\text { (3) }\end{array}$ \\
\hline Argentina (AR) & 30,24 & $81(10)$ & $\mathrm{HI}$ & 48(2) & 0,56 & $98(12)$ \\
\hline $\begin{array}{l}\text { Bolivia (Estado } \\
\text { Plurinacional de) (BO) }\end{array}$ & 25,24 & $109(17)$ & LM & 105(18) & 0,59 & 89(9) \\
\hline Brasil (BR) & 33,19 & $69(7)$ & UM & 66(9) & 0,55 & $100(13)$ \\
\hline Chile (CL) & 58,41 & $44(1)$ & $H$ & $40(4)$ & 0,59 & $91(10)$ \\
\hline Colombia (CO) & 34,16 & 63(5) & UM & $63(7)$ & 0,56 & $96(11)$ \\
\hline Costa Rica (CR) & 38,40 & 45(2) & UM & $54(5)$ & 0,71 & $50(1)$ \\
\hline $\begin{array}{l}\text { República } \\
\text { Dominicana (RD) }\end{array}$ & 30,55 & 76(9) & UM & $70(11)$ & 0,62 & $82(7)$ \\
\hline Ecuador (EC) & 27,11 & $100(14)$ & UM & $82(14)$ & 0,60 & 87(8) \\
\hline El Salvador (ES) & 26,56 & 104(16) & LM & 101(17) & 0,48 & $113(17)$ \\
\hline Guatemala (GT) & 27,30 & $97(13)$ & LM & $97(15)$ & 0,62 & $79(5)$ \\
\hline Honduras (HN) & 26,94 & 101(15) & LM & $98(16)$ & 0,53 & $105(15)$ \\
\hline Jamaica (JM) & 28,97 & $89(11)$ & UM & $77(12)$ & 0,53 & 104(14) \\
\hline México (MX) & 34,56 & 61(3) & UM & $63(6)$ & 0,63 & $76(3)$ \\
\hline Nicaragua (NI) & 23,06 & 116(18) & LM & 102(19) & 0,41 & 120(19) \\
\hline Panamá (PA) & 33,49 & 68(6) & UM & 65(8) & 0,66 & 61(2) \\
\hline Paraguay (PY) & 28,20 & $94(12)$ & UM & $81(13)$ & 0,62 & $77(4)$ \\
\hline Perú (PE) & 32,51 & 71(8) & UM & $68(10)$ & 0,51 & 109(16) \\
\hline Uruguay (UR) & 34,28 & 62(4) & $H$ & $45(1)$ & 0,62 & $81(6)$ \\
\hline $\begin{array}{l}\text { Venezuela (República } \\
\text { Bolivariana de) (VE) }\end{array}$ & 22,32 & 120(19) & - & 49(3) & 0,46 & 114(18) \\
\hline
\end{tabular}

Fuente: Elaboración propia, sobre la base de Universidad de Cornell/INSEAD/Organización Mundial de la Propiedad Intelectual (OMPI), The Global Innovation Index 2016: Winning with Global Innovation, Ginebra, 2016.

Nota: LM: ingreso medio-bajo; UM: ingreso medio-alto; HI: ingreso alto. En las columnas 3, 5 y 7 , el primer valor corresponde a la posición del país a nivel mundial y el segundo valor (entre paréntesis) se refiere a la posición del país con respecto a los demás países de América Latina y el Caribe.

Desde una perspectiva global, la correlación entre la clasificación de ingreso y la razón de eficiencia es del 29,84\%, mientras que la correlación entre el ingreso y el índice general es del 68,81\% (véase el cuadro 2). Esto permite inferir que existe una asociación notable entre el nivel de ingresos de la población y el nivel de insumos para la innovación (que tienen un peso importante en el índice general). La relación, no obstante, no es tan sólida entre la eficiencia en el uso de estos insumos y el nivel de riqueza en la economía. Por ejemplo, economías como las de Bolivia (Estado Plurinacional de), el Ecuador, Guatemala, Panamá, el Paraguay y Venezuela (República Bolivariana de) mostraron una mejor posición en términos de aprovechamiento de insumos (razón de eficiencia) que la conseguida en el índice general, tanto a nivel mundial como regional.

En el gráfico 1 se muestra un conjunto de formas radiales correspondientes a diferentes medidas del Índice Global de Innovación asociadas con la creación de entornos favorables para la 
innovación. De acuerdo con estos datos, más allá de que la puntuación media de los productos para la innovación sea mucho menor que la de los insumos, es notoria la falta de paralelismo entre las espirales que forman ambas puntuaciones en términos de países ${ }^{11}$.

\section{Gráfico 1}

América Latina y el Caribe: representaciones radiales de diferentes medidas del Índice Global de Innovación (IGI)

A. Subíndices de entrada $(-)$ y salida $(-)$

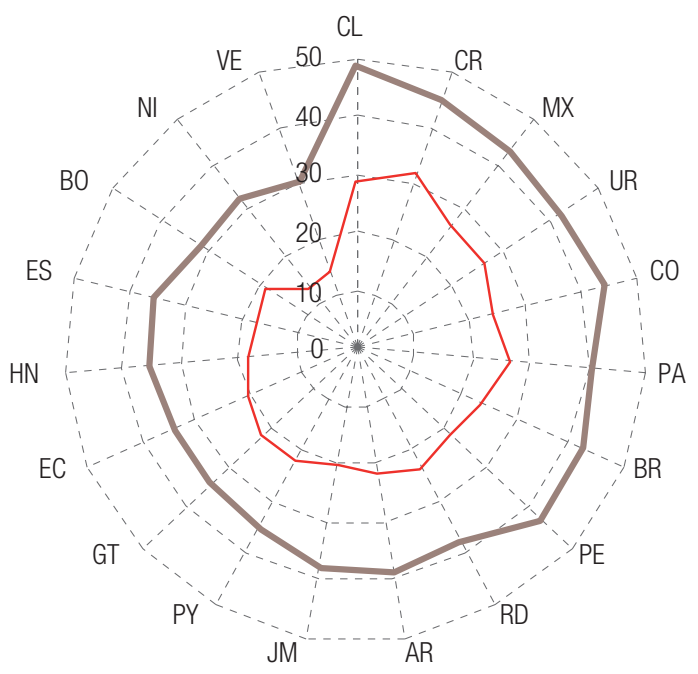

\section{B. Razón de eficiencia}

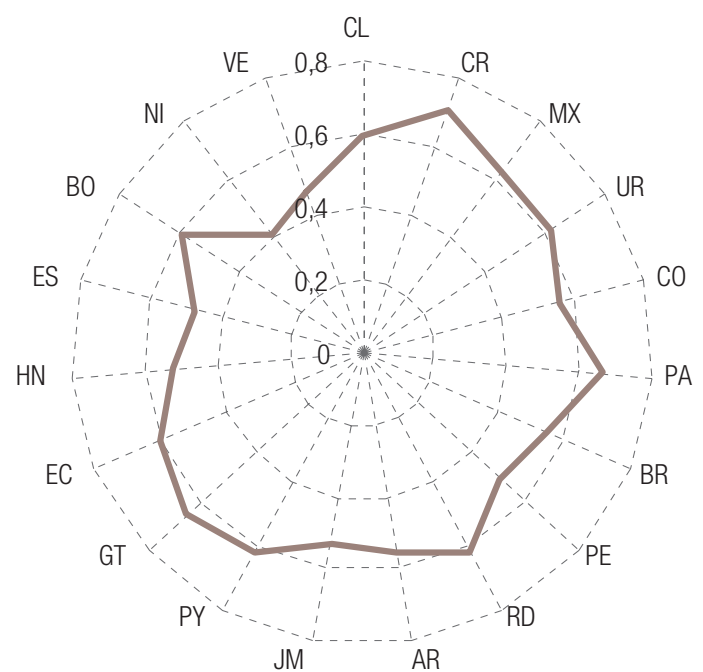

Fuente: Elaboración propia, sobre la base de Universidad de Cornell/INSEAD/Organización Mundial de la Propiedad Intelectual (OMPI), The Global Innovation Index 2016: Winning with Global Innovation, Ginebra, 2016.

Nota: Los países se presentan en orden descendente con respecto a su posición en el índice general y Chile (CL) ocupa la primera posición. La nomenclatura empleada en este gráfico se explica en el cuadro 2.

En este sentido, Bolivia (Estado Plurinacional de) y Venezuela (República Bolivariana de), ya descontando que están a la cola en el índice general para América Latina y el Caribe, presentan una puntuación en el subíndice de entrada por debajo de lo que se esperaría en una espiral de tendencia descendente. En el Brasil, Chile, Colombia, El Salvador o el Perú, por el contrario, el nivel de salidas no se corresponde con la dotación de insumos (véase el gráfico 1.A), por lo que ninguno de estos países logra superar la décima posición en la razón de eficiencia (véase el cuadro 2).

Por otra parte, se encontró una marcada variabilidad en las posiciones de las economías, medida como la diferencia entre el lugar que ocupan en un indicador y su posición en el índice general. Se destaca que el 22,44\% de las posiciones de los países de América Latina y el Caribe para los diferentes indicadores de entrada fueron peores que la de la economía número 100 en el mundo y un $17,21 \%$ de estas fueron mejores que la de la economía número 50. Cuando se revisan los productos de la innovación, no obstante, se observa que el 28,91\% de los resultados estuvieron más allá de la economía número 100 en el ámbito global y tan solo el 8,77\% de ellos figuraron entre las primeras 50 economías. Esto muestra que la posición relativa de los indicadores empeora en América Latina y el Caribe cuando se pasa de los insumos a los productos.

\footnotetext{
${ }^{11}$ Cabe mencionar que la diferencia en la puntuación no es tan acentuada en algunas otras regiones del mundo.
} 


\section{Relaciones entre insumos y productos en los países de América Latina y el Caribe ${ }^{12}$}

En principio, cabría esperar una relación positiva entre las puntuaciones de los indicadores de entrada y salida para el conjunto de países tratados, lo cual suele cumplirse ${ }^{13}$. La evidencia muestra una correlación positiva "uno a uno" entre los tres indicadores del pilar institucional y los seis indicadores de salida ${ }^{14}$. Los países con mayor presencia en las fronteras eficientes son el Brasil (media), Costa Rica (media-superior) y Chile (superior), todos ellos ubicándose por encima de la media de ingreso en América Latina y el Caribe ${ }^{15}$.

También se encuentra una correlación positiva - aunque incipiente en algunos casos - entre los niveles de los insumos de capital humano e investigación y cada uno de los productos, excepto en el caso de la educación terciaria e I+D y la generación de activos intangibles, que es negativa. Esto es preocupante, pues muestra que la inversión en educación superior e investigación en América Latina y el Caribe no conduce a un desarrollo suficiente de su capital intelectual o a la creación de valor de sus marcas, diseños o patentes ${ }^{16}$. Los países que suelen conformar la frontera en estas relaciones son, nuevamente, el Brasil (media-inferior), Costa Rica (media-superior) y Chile (superior). Se presenta el caso atípico del Brasil con el peor indicador en educación terciaria en la región y uno de los niveles más altos en creación e impacto del conocimiento. A diferencia de lo que ocurre en América Latina y el Caribe, en el caso de Europa existe una relación positiva entre educación terciaria y activos intangibles. Lo expuesto pone de manifiesto que no será suficiente contar con altos niveles de insumos para reducir las brechas económicas y sociales que existen entre América Latina y el Caribe y otras regiones del mundo.

Por otra parte, existe una correlación positiva entre todos los elementos del pilar infraestructura y cada uno de los productos. Los países con mayor presencia en la frontera eficiente son Guatemala (media-inferior), Costa Rica (media-superior), Colombia (superior) y Chile (superior). En materia de TIC, llama la atención el caso de Costa Rica, que aparece por debajo de la línea de tendencia en cuatro de los productos, aunque en el resto forma parte de la frontera eficiente. También es llamativa la forma en que Panamá, con el mejor indicador de infraestructura de América Latina y el Caribe, presenta un desempeño modesto como resultado de sus bajos niveles de salida. Un caso inverso es el de Guatemala, ya que, con el peor indicador de infraestructura, logra situarse en las fronteras eficientes, aun sin niveles sobresalientes de salida.

Los indicadores del pilar sofisticación del mercado son los que menos relaciones positivas muestran cuando se trasponen con los productos (un 55,56\% de correlaciones positivas). En este sentido, la inversión es el insumo que menos impacto tiene en el desarrollo de la innovación en América Latina y el Caribe, aunque tampoco resulta clara la contribución del crédito a la creación y difusión del conocimiento, así como a la creatividad digital ${ }^{17}$. Los países que suelen conformar la frontera son Costa Rica (media-superior), Chile (superior) y Colombia (superior). México está por debajo de la línea de tendencia en tres de los seis productos cuando se habla de competencia comercial y escala de

\footnotetext{
12 Se debe tomar en cuenta que tanto los productos como los insumos están referidos a un mismo período de tiempo.

${ }^{13}$ De hecho, existe una correlación del 40,92\% entre el nivel de los insumos y la razón de eficiencia que avala parcialmente esta conjetura.

${ }^{14}$ En estos términos habría que entender también la construcción de las fronteras eficientes a las que se hace referencia en esta subsección.

${ }^{15}$ Se descarta del análisis la parte inferior de la frontera eficiente al estar asociada con valores incipientes de los insumos.

${ }^{16}$ En Europa existe una relación positiva entre estos insumos y los activos intangibles. Incluso África Septentrional y Asia muestran una correlación ligeramente positiva entre estos indicadores (aunque en este caso las relaciones con la mayoría del resto de los productos no son positivas).

${ }^{17}$ A diferencia de lo que ocurre en América Latina y el Caribe, en Europa se da una relación positiva entre el insumo de inversión y los productos, con excepción del caso del impacto del conocimiento.
} 
mercado, indicador en el que ocupa la primera posición de América Latina y el Caribe. Bolivia (Estado Plurinacional de) y el Perú, por su parte, tienen los niveles más altos en el factor crédito, pero están lejos de alcanzar los niveles de salida necesarios para formar parte de la frontera eficiente.

En materia de sofisticación de negocios, aunque la absorción del conocimiento se asocia positivamente con todos los productos, en el caso de los trabajadores del conocimiento y los vínculos de innovación este signo se observa solo con tres de estos. Ello podría constituir un comportamiento particular de América Latina y el Caribe relacionado con este pilar si se comprueba que la absorción del conocimiento (entendido como un insumo) se está aprovechando como alternativa de desarrollo ante la debilidad que para ello muestran otros insumos. Los países que suelen conformar la frontera son Chile (superior), Costa Rica (superior) y el Uruguay (superior).

Por último, es importante destacar que las relaciones negativas entre los indicadores de entrada y salida harían sospechar sobre si realmente los primeros propician la ocurrencia de los segundos. Alrededor del $25 \%$ de las relaciones individuales entre estos pilares presentaron una tendencia negativa para América Latina y el Caribe (véase el gráfico 2).

Gráfico 2

Algunas relaciones entre insumos y productos con tendencia negativa
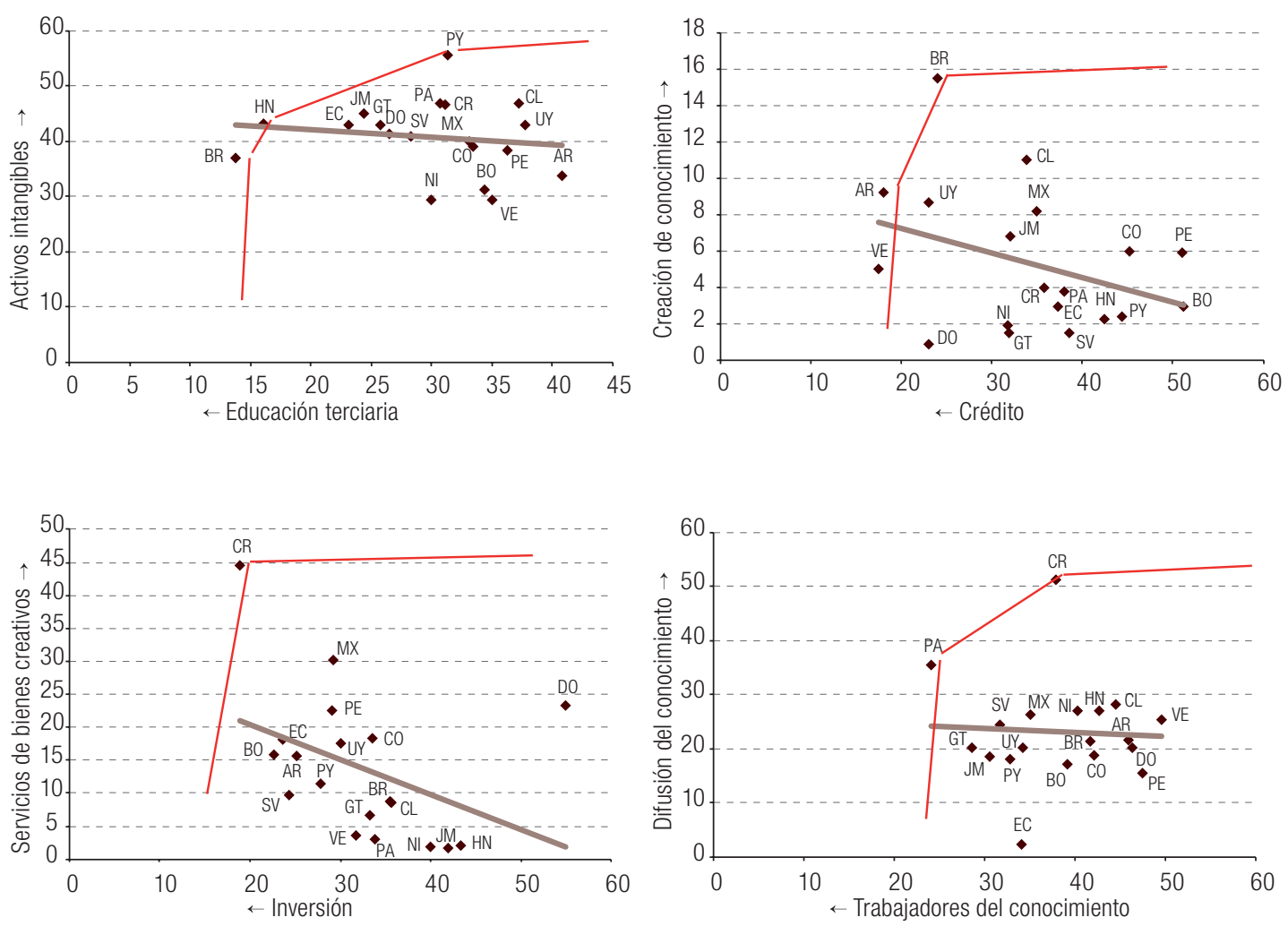

Fuente: Elaboración propia, sobre la base de Universidad de Cornell/INSEAD/Organización Mundial de la Propiedad Intelectual (OMPI), The Global Innovation Index 2016: Winning with Global Innovation, Ginebra, 2016.

Nota: La línea de guiones representa una hipotética frontera eficiente entre las variables graficadas siguiendo la premisa "más productos con menos insumos". La línea continua muestra la tendencia de los datos. 


\section{Metodología}

La medición de la eficiencia consiste en evaluar la actuación de los múltiples indicadores de una organización en la búsqueda de un óptimo (Álvarez, 2001). Partiendo de la premisa anterior, una aproximación al nivel de eficiencia resultaría de comparar aquello que hace la unidad de decisión con lo que debería haber hecho para maximizar su beneficio. Es probable, sin embargo, que no se cuente con información completa acerca del contexto en el cual se desenvuelven las unidades de decisión y, por lo tanto, acerca de cuál es el máximo beneficio potencial de cada una. La mejor manera de superar esta falta de información es realizar una comparación entre la unidad de decisión en cuestión y aquellas que posean el mejor desempeño y que, al mismo tiempo, tengan características similares a la unidad de referencia.

Partiendo de la definición de eficiencia planteada anteriormente, es importante ahondar en la distinción entre eficiencia técnica y asignativa. La primera se consigue cuando resulta tecnológicamente imposible aumentar algún producto o reducir algún insumo a costa de otro (optimalidad de Pareto), mientras que la segunda (llamada también "eficiencia en precios") supone minimizar el desperdicio de recursos (Navarro y Torres, 2006), lo que es equivalente a elegir la opción más barata entre las combinaciones de insumos y productos eficientes. Dado que este estudio supone que los agentes tienen información asimétrica y muestran cierto nivel de aversión al riesgo, se centrará en el análisis de la eficiencia técnica.

El método de análisis envolvente de datos permite estudiar la eficiencia individual relativa de un conjunto de unidades productivas en relación con el comportamiento de otras similares, a partir de la construcción de una frontera eficiente (real y no ideal, relativa y no absoluta). Esto se lleva a cabo mediante aproximaciones no paramétricas que permiten incorporar supuestos sobre las prioridades de la tecnología de producción y los planes de producción realizables (Martínez, de Miguel y Murias, 2005; Restrepo y Villegas, 2007). Según Quindós, Rubiera y Vicente (2003), el método de análisis envolvente de datos posee dos fortalezas: su grado de estandarización y la posibilidad de considerar múltiples insumos y productos.

En este trabajo se hará uso de elementos tanto del análisis envolvente de datos según BankerCharnes-Cooper (BCC), quienes se orientan a los productos, como de la versión original de CharnesCooper-Rhodes (CCR), con orientación a los insumos (Banker y otros, 1990). Sin embargo, el segundo método tendrá más peso debido a que permite identificar diferencias en las escalas productivas de las unidades de decisión, a que su modelaje presenta menos restricciones y a que resulta más conveniente cuando no son explícitos los procesos y mecanismos por los cuales los insumos se transforman en productos, como en este caso.

En un contexto tradicional de estimación de eficiencia, el análisis envolvente de datos sugiere que, a partir de un proceso productivo en el que se utilizan $p$ insumos $\left(x_{1}, x_{2}, \ldots, x_{p}\right)$ para producir $q$ productos $\left(y_{1}, y_{2}, \ldots, y_{q}\right)$ y donde participan $n$ unidades productivas, la eficiencia técnica de una determinada unidad 0 puede estimarse a través de la siguiente expresión:

$$
\max _{u_{r}, v_{i}} \frac{\hat{A}_{r=1}^{q} u_{r} y_{r 0}}{\hat{A}_{i=1}^{p} v_{i} x_{i 0}}
$$

sujeta a

$$
\frac{\sum_{r=1}^{q} u_{r} y_{r j}}{\sum_{i=1}^{p} v_{i} x_{i j}} \leq 1
$$


donde $j=1, \ldots, n, r=1, \ldots, q$ e $i=1, \ldots, p$. Además $u_{i}, v_{i} \geq e$, donde $e$ representa un valor infinitesimalmente pequeño pero mayor que cero. La idea que subyace es la de maximizar una especie de índice de productividad total de factores (unidades de salida producidas por cada unidad de entrada empleada) para cada unidad de decisión. En el numerador del índice se resumen todos los productos en un único producto virtual, al igual que en el denominador, donde un único insumo virtual recoge todos los factores empleados en el proceso productivo. Para estos agregados no se utiliza un sistema de precios convencional, sino un conjunto de ponderaciones $\left(u_{1}, \ldots, u_{q}, v_{1}, \ldots, v_{p}\right)$ - cuyo valor se pretende encontrar -, que deben hacer máxima la razón para cada unidad de decisión, manteniendo, al mismo tiempo, por debajo de uno las razones de las demás.

Además de este resultado básico, el análisis envolvente de datos proporciona información adicional tal como los insumos y productos virtuales, es decir, la aportación de cada factor al índice de eficiencia estimado, o los niveles de insumos y productos que convertirían en eficiente a una unidad que no lo es. Uno de los principales atractivos de esta técnica es que permite equilibrar los elementos objetivos y subjetivos del aspecto a investigar, facilitando la identificación de las fortalezas y debilidades de cada una de las unidades de decisión que se comparan.

El análisis envolvente de datos se ha empleado en la medición y comparación de la eficiencia en sectores considerados clave para el desarrollo de los países (tales como el educativo, bancario, de salud, de innovación y otros). En las últimas décadas han surgido nuevas aplicaciones de este análisis en contextos distintos al estrictamente productivo, relevantes sobre todo para el análisis regional del desarrollo. Ejemplos de ello son la estimación del índice de bienestar social y calidad de vida (Hashimoto y Kodama, 1997) o del índice de bienestar económico a nivel provincial (Martínez, de Miguel y Murias, 2005).

No obstante, el método también presenta limitaciones y riesgos. Una de las principales desventajas del análisis envolvente de datos radica en el reto de definir adecuadamente los insumos y los productos (en este trabajo dicha definición se basa en una clasificación externa). También suele ser complejo poder cubrir la exigencia de la homogeneidad de las unidades sometidas a análisis y la de los usos que estas hacen de sus insumos y productos. En este artículo se supondrá a priori que los productos están asociados a los niveles de los insumos y que las decisiones de las unidades de decisión (países) acerca de los segundos tienen un impacto de corto plazo sobre los niveles de los primeros. Es importante mencionar, a su vez, que los insumos considerados tienen asociados costos tangibles e intangibles para las economías nacionales, cuyo financiamiento representa un costo de oportunidad que no se considera en el análisis.

Por otra parte, la magnitud y calidad de los productos de innovación están parcialmente determinadas por la estructura regional específica de las unidades de medición y las diferencias entre los sectores que las componen. Si bien es cierto que tomar en cuenta estas diferencias hace que los resultados sean científicamente más precisos (Broekel, Rogge y Brenner, 2018), también requiere de una mayor cantidad de datos y de conocimientos acerca del desempeño relativo de las economías, y con ello surge el riesgo de aparición de juicios de valor. Dado el carácter exploratorio del presente trabajo y las ventajas que presenta el uso de medidas generales en materia de discusión de políticas, no se utilizan ponderadores para diferenciar sectores o unidades de medición. 


\section{Resultados}

\section{Resultados de eficiencia}

En el marco de una orientación a los insumos, esto es, el cálculo de la máxima reducción en los insumos para lograr un determinado nivel de productos, se pretende encontrar la eficiencia en la generación de un entorno innovador en los países de América Latina y el Caribe. La diferencia entre el valor máximo posible de eficiencia (100\%) y el valor observado en una unidad de medición representa el tamaño de su ineficiencia (Coelli y otros, 2003) ${ }^{18}$. En la medida en que el valor se acerque al máximo posible, la unidad de decisión estará más cerca de la frontera eficiente. En el cuadro 3 se presentan los resultados de eficiencia en la gestión de los insumos de cada pilar con relación al nivel de productos que generan.

\section{Cuadro 3}

Resultados de eficiencia por pilar, 2016

(En porcentajes)

\begin{tabular}{|c|c|c|c|c|c|}
\hline \multirow{2}{*}{ Pilar } & \multirow{2}{*}{ País $^{\mathrm{a}}$} & \multicolumn{3}{|c|}{ Eficiencia técnica: } & \multirow{2}{*}{ Retornos } \\
\hline & & global & pura & de escala & \\
\hline \multirow{9}{*}{ Institucional } & Chile & 95,05 & 100 & 95,05 & Decreciente \\
\hline & República Dominicana & 97,83 & 100 & 97,83 & Decreciente \\
\hline & El Salvador & 68,02 & 71,13 & 95,63 & Decreciente \\
\hline & Honduras & 97,50 & 100 & 97,50 & Decreciente \\
\hline & Jamaica & 72,79 & 72,80 & 99,99 & Creciente \\
\hline & Nicaragua & 65,40 & 67,76 & 96,52 & Decreciente \\
\hline & Perú & 93,82 & 94,77 & 99,00 & Creciente \\
\hline & Uruguay & 99,98 & 100 & 99,98 & Decreciente \\
\hline & Resto de países & 100 & 100 & 100 & Constante \\
\hline \multirow{6}{*}{$\begin{array}{l}\text { Capital humano } \\
\text { e investigación }\end{array}$} & Argentina & 79,01 & 80,22 & 98,60 & Creciente \\
\hline & Bolivia (Estado Plurinacional de) & 72,02 & 73,58 & 97,88 & Decreciente \\
\hline & Honduras & 68,49 & 100 & 68,49 & Decreciente \\
\hline & Paraguay & 93,42 & 100 & 93,42 & Decreciente \\
\hline & $\begin{array}{l}\text { Venezuela (República } \\
\text { Bolivariana de) }\end{array}$ & 53,97 & 55,66 & 96,96 & Creciente \\
\hline & Resto de países & 100 & 100 & 100 & Constante \\
\hline \multirow{6}{*}{ Infraestructura } & Colombia & 98,39 & 100 & 98,39 & Decreciente \\
\hline & Ecuador & 99,81 & 100 & 99,81 & Decreciente \\
\hline & El Salvador & 89,82 & 94,25 & 95,30 & Creciente \\
\hline & Perú & 90,72 & 92,57 & 98,00 & Creciente \\
\hline & $\begin{array}{l}\text { Venezuela (República } \\
\text { Bolivariana de) }\end{array}$ & 80,28 & 98,07 & 81,86 & Creciente \\
\hline & Resto de países & 100 & 100 & 100 & Constante \\
\hline
\end{tabular}

\footnotetext{
${ }^{18}$ La eficiencia técnica global depende de la ocurrencia de la eficiencia técnica pura (aspectos operacionales) y la eficiencia técnica de escala (aspectos dimensionales). La eficiencia técnica de escala se calcula por el cociente de la eficiencia técnica global y la eficiencia técnica pura.
} 
Cuadro 3 (conclusión)

\begin{tabular}{|c|c|c|c|c|c|}
\hline \multirow{2}{*}{ Pilar } & \multirow{2}{*}{ País ${ }^{\mathrm{a}}$} & \multicolumn{3}{|c|}{ Eficiencia técnica: } & \multirow{2}{*}{ Retornos } \\
\hline & & global & pura & de escala & \\
\hline \multirow{8}{*}{ Sofisticación del mercado } & Chile & 98,12 & 100 & 98,12 & Decreciente \\
\hline & Colombia & 90,57 & 100 & 90,57 & Decreciente \\
\hline & El Salvador & 80,16 & 95,82 & 83,66 & Creciente \\
\hline & Guatemala & 82,21 & 84,44 & 97,36 & Creciente \\
\hline & Honduras & 96,92 & 100 & 96,92 & Creciente \\
\hline & Nicaragua & 68,08 & 93,87 & 72,53 & Creciente \\
\hline & Perú & 77,44 & 81,63 & 94,87 & Creciente \\
\hline & Resto de países & 100 & 100 & 100 & Constante \\
\hline \multirow{6}{*}{ Sofisticación de negocios } & Chile & 96,70 & 100 & 96,70 & Decreciente \\
\hline & El Salvador & 96,94 & 100 & 96,94 & Creciente \\
\hline & Honduras & 86,20 & 88,09 & 97,85 & Decreciente \\
\hline & Nicaragua & 81,82 & 88,35 & 92,60 & Creciente \\
\hline & Perú & 89,74 & 92,59 & 96,93 & Creciente \\
\hline & Resto de países & 100 & 100 & 100 & Constante \\
\hline
\end{tabular}

Fuente: Elaboración propia.

a Solo se incluyen los países que presentaron algún grado de ineficiencia técnica global en cada pilar.

De acuerdo con el cuadro 3, los pilares donde se presenta un mayor número de países con ineficiencias en términos globales y de escala son el institucional y el de sofisticación del mercado. Aunque, como ya se señaló, el segundo muestra una mala asociación con los productos, las ineficiencias en los mecanismos institucionales, muy asociados con estos, sí representan una pérdida de oportunidad en el desarrollo de la región.

En particular, el pilar infraestructura presenta el mejor valor medio en términos de eficiencia técnica global y eficiencia técnica pura. Países como El Salvador, Honduras y el Perú muestran ineficiencias en cuatro pilares, mientras que Chile y Nicaragua revelan ineficiencias en tres pilares. El Brasil, Costa Rica, México y Panamá son los únicos países que no aparecen con ineficiencias en ningún pilar. Por otra parte, Chile, Colombia y Honduras suelen mostrar retornos decrecientes a escala, por lo que su producto es inferior al esperado, en tanto que El Salvador, el Perú y Venezuela (República Bolivariana de) por lo general presentan una dimensión superior a la óptima. Chile y Honduras también son los países que con más frecuencia muestran un buen desempeño operacional, aun con ineficiencias en la escala ${ }^{19}$.

El buen desempeño de Chile en términos de eficiencia pura deja de manifiesto que las malas calificaciones que recibe este país en algunas mediciones se deben a su peso en los aspectos de escala y rendimiento. Es por ello también que tres de los cuatro países que no muestran ineficiencias ocupan las tres primeras posiciones regionales en la razón de eficiencia (que se basa en el tipo de rendimientos), mientras que los que aparecen con ineficiencias en cuatro pilares están más allá de la decimoquinta posición regional en este indicador.

\footnotetext{
${ }^{19}$ Finalmente, las economías con peores niveles de eficiencia global fueron El Salvador, Honduras, Nicaragua y Venezuela (República Bolivariana de). Este resultado se debe, sobre todo, a la debilidad de sus instituciones y la formación de capital humano.
} 


\section{Resultados de holguras y contribuciones relativas}

El análisis de holguras da cuenta del sentido y la magnitud en que deben ajustarse los niveles de los indicadores en las unidades de decisión para transformar un país débilmente eficiente en uno eficiente. Una holgura en los productos indica la necesidad de que estos se incrementen, mientras que una holgura en los insumos es señal de un exceso de ellos, de acuerdo con la dimensión sugerida por el valor conjunto de sus indicadores (Lo, Chien y Lin, 2001). En el cuadro 4 se observa, por ejemplo, que el Perú requiere modificaciones en sus indicadores de entrada y salida en cuatro pilares para acercarse a la frontera de la eficiencia ${ }^{20}$. Al Perú le siguen El Salvador y Nicaragua, que requieren modificaciones en tres pilares, y Venezuela (República Bolivariana de), que necesita cambios en dos pilares.

\section{Cuadro 4}

Resultados de holguras por pilar, 2016

(En porcentajes)

\begin{tabular}{|c|c|c|c|c|c|c|c|c|c|c|}
\hline \multirow{2}{*}{ Pilar } & \multirow{2}{*}{ País } & \multicolumn{3}{|c|}{ Holguras de insumos } & \multicolumn{6}{|c|}{ Holguras de productos } \\
\hline & & IP1 & IP2 & IP3 & OP1 & $\mathrm{OP} 2$ & OP3 & OP4 & OP5 & OP6 \\
\hline \multirow{4}{*}{ Institucional } & El Salvador & 3,30 & 17,63 & - & 2,42 & 3,00 & 0,14 & - & - & 2,01 \\
\hline & Jamaica & - & 1,65 & - & - & - & 1,04 & - & 8,61 & 3,12 \\
\hline & Nicaragua & - & 35,84 & 11,48 & 3,03 & 0,88 & - & 1,04 & 4,51 & 3,75 \\
\hline & Perú & - & 15,49 & - & 1,07 & 2,23 & 8,95 & - & - & - \\
\hline \multirow{3}{*}{$\begin{array}{l}\text { Capital humano } \\
\text { e investigación }\end{array}$} & Argentina & - & - & 1,75 & - & 11,16 & - & 7,95 & - & - \\
\hline & $\begin{array}{l}\text { Bolivia (Estado } \\
\text { Plurinacional de) }\end{array}$ & 1,90 & - & - & - & - & - & 11,39 & 0,50 & 2,02 \\
\hline & $\begin{array}{l}\text { Venezuela (República } \\
\text { Bolivariana de) }\end{array}$ & - & 13,44 & 5,68 & - & 10,06 & 1,10 & 3,35 & - & - \\
\hline \multirow{3}{*}{ Infraestructura } & El Salvador & 15,86 & - & - & 0,46 & 21,50 & - & 1,92 & - & 0,44 \\
\hline & Perú & - & - & 4,46 & - & 3,85 & 12,90 & 3,11 & - & - \\
\hline & $\begin{array}{l}\text { Venezuela (República } \\
\text { Bolivariana de) }\end{array}$ & 11,49 & - & - & - & 17,86 & - & 13,67 & - & - \\
\hline \multirow{4}{*}{ Sofisticación del mercado } & El Salvador & - & - & - & 2,25 & 14,98 & 9,62 & - & 15,91 & 3,26 \\
\hline & Guatemala & - & - & - & 5,88 & - & 3,92 & - & 13,75 & 13,65 \\
\hline & Nicaragua & - & 0,87 & - & 1,80 & 5,43 & - & 7,05 & 2,61 & 1,72 \\
\hline & Perú & 10,20 & - & - & - & 1,94 & 20,68 & 5,93 & 9,10 & 3,99 \\
\hline \multirow{3}{*}{ Sofisticación de negocios } & Honduras & - & 12,07 & - & 1,49 & 1,21 & - & - & 7,14 & 6,28 \\
\hline & Nicaragua & - & - & - & 3,43 & 19,12 & - & 11,27 & 5,66 & 11,83 \\
\hline & Perú & 5,71 & - & - & 1,12 & 3,55 & 3,70 & - & - & 0,30 \\
\hline
\end{tabular}

Fuente: Elaboración propia.

Nota: Los insumos representados por IP1, IP2 e IP3 son diferentes en función del pilar. Para institucional, IP1: Ambiente político; IP2: Entorno regulatorio; IP3: Ambiente de negocios. Para capital humano e investigación, IP1: Educación secundaria; IP2: Educación terciaria; IP3: investigación y desarrollo (I+D). Para infraestructura, IP1: tecnologías de la información y las comunicaciones (TIC); IP2: Infraestructura; IP3: Sostenibilidad ecológica. Para sofisticación del mercado, IP1: Crédito; IP2: Inversión; IP3: Competencia comercial y escala de mercado. Para sofisticación de negocios, IP1: Trabajadores del conocimiento; IP2: Vínculos de innovación; IP3: Absorción del conocimiento. OP1: Creación de conocimiento. OP2: Impacto del conocimiento. OP3: Difusión del conocimiento. OP4: Activos intangibles. OP5: Servicios de bienes creativos. OP6: Creatividad digital.

\footnotetext{
20 Sin embargo, los resultados medios no sitúan al Perú entre las cuatro economías con peor desempeño.
} 
El indicador de salida que más frecuentemente muestra holgura es impacto del conocimiento, seguido de creatividad digital (para los que ya se había detectado alguna disociación con insumos como el crédito). En el primero, países como El Salvador, Nicaragua y Venezuela (República Bolivariana de) requieren hacer incrementos de cerca del 20\% en algunos casos. El Perú manifiesta problemas en difusión del conocimiento. No obstante, es Nicaragua el país más deficitario en términos de indicadores de salida, ya que requiere ajustes en casi todos ellos ${ }^{21}$. Estas problemáticas se suman a otras ya identificadas (por ejemplo, en la generación de activos intangibles) cuando se analizaron las relaciones entre insumos y productos, en las que incluso se llegaron a encontrar asociaciones negativas. Cabe destacar también que los niveles de infraestructura, absorción del conocimiento y competencia comercial y escala de mercado se emplean de manera óptima en la mejora de la eficiencia en los 19 países de la región, lo que refuerza la conjetura de que los principales problemas regionales radican en los aspectos institucionales y de capital humano e investigación.

En contraste con el análisis de holguras, la contribución relativa de los indicadores indica los puntos fuertes y débiles de un país en la gestión para la consecución de ciertos objetivos. Un país que presente una ventaja relativa en un indicador tenderá a obtener una mayor contribución a partir de este (Martínez y Murias, 2011). Se encontró que el ambiente de negocios fue el indicador de mayor contribución media a la eficiencia de la innovación en América Latina y el Caribe en materia de aspectos institucionales, mientras que el entorno regulatorio fue la entrada que menos contribuyó a este objetivo dentro del pilar institucional.

Con referencia al capital humano e investigación, la educación secundaria fue el indicador en el que descansó primordialmente la eficiencia en innovación, mientras que la I+D fue la entrada que menos contribuyó. En el caso del pilar infraestructura, las TIC fueron el indicador de mayor contribución - lo que es congruente con lo sugerido por Bárcena (2008) -, aunque el Brasil y Colombia prefirieron dar más peso a la infraestructura para sacar el mejor provecho del potencial innovador, mientras que Costa Rica, el Ecuador, El Salvador y Venezuela (República Bolivariana de) otorgaron más peso a la sostenibilidad ecológica.

Los indicadores del pilar sofisticación del mercado contribuyeron de forma muy equitativa al fortalecimiento de la innovación (entre un 30\% y un 35\% cada uno), en promedio, para los países de América Latina y el Caribe. En lo que se refiere a la sofisticación de negocios, trabajadores del conocimiento fue el indicador en el que más se apoyaron los países de la región y vínculos de innovación fue el indicador que menos aportó. Esto es evidencia de que los países de la región están teniendo problemas para aprovechar el indicador de mayor impacto potencial del pilar (absorción del conocimiento) para incrementar el nivel de los productos.

\section{Comparativo internacional}

Con la finalidad de situar a América Latina y el Caribe en el contexto internacional, en el cuadro 5 se presenta el diferencial porcentual de esta región respecto de las otras regiones del mundo para cada uno de los pilares de entrada y salida, además de la razón de eficiencia. Se encontró que América del Norte saca el mayor diferencial positivo respecto de América Latina y el Caribe en todos los pilares, lo que hasta cierto punto era previsible dado que esta región ocupa la primera posición en 17 de los 21 indicadores que los componen (Europa tiene las otras cuatro primeras posiciones).

\footnotetext{
${ }^{21}$ Incluso presenta un entorno regulatorio (pilar institucional) altamente desaprovechado para su nivel de producto, al igual que El Salvador y el Perú.
} 


\section{Cuadro 5}

Diferencias porcentuales de las puntuaciones de los pilares de innovación y la razón de eficiencia entre las distintas regiones del mundo y América Latina y el Caribe, 2016

(En porcentajes)

\begin{tabular}{lrrrrrrrr}
\hline Región & P1 & P2 & P3 & P4 & P5 & P6 & P7 & RE \\
\hline América del Norte & 67,58 & 109,17 & 54,56 & 89,41 & 60,77 & 169,38 & 89,19 & 28,07 \\
\hline Europa & 43,59 & 77,75 & 31,07 & 17,75 & 31,26 & 107,68 & 67,15 & 31,58 \\
\hline Asia Sudoriental, Asia Oriental y Oceanía & 31,68 & 63,52 & 26,76 & 34,59 & 34,87 & 99,34 & 43,66 & 24,56 \\
\hline África Septentrional y Asia Occidental & 14,21 & 21,80 & 10,61 & $-0,43$ & $-12,54$ & 33,89 & 10,08 & 10,53 \\
\hline Asia Central y Asia Meridional & $-6,54$ & $-5,55$ & $-12,76$ & $-3,95$ & $-16,22$ & 10,12 & $-20,62$ & 3,51 \\
\hline África Subsahariana & $-0,96$ & $-31,61$ & $-29,72$ & $-15,08$ & $-10,43$ & 1,77 & $-27,18$ & 1,75 \\
\hline
\end{tabular}

Fuente: Elaboración propia, sobre la base de Universidad de Cornell/INSEAD/Organización Mundial de la Propiedad Intelectual (OMPI), The Global Innovation Index 2016: Winning with Global Innovation, Ginebra, 2016.

Nota: Véase la identificación de los pilares en el cuadro 1. RE: razón de eficiencia.

El cuadro 5 resulta también muy revelador pues en él se muestra que, de acuerdo con el puntaje de los pilares, únicamente las regiones de Asia Central y Asia Meridional y África Subsahariana presentan niveles por debajo de los de América Latina y el Caribe en la mayoría de los pilares y que ninguna región del mundo exhibe un comportamiento generalizado inferior al de la citada región. Asimismo, es destacable mencionar que en el caso del pilar de salida conocimiento y tecnología (P6) -e incluso en términos de la razón de eficiencia - no existen regiones con puntaje tan bajo como el de América Latina y el Caribe 22 .

\section{Conclusiones}

Los países de América Latina y el Caribe tratan de adaptarse a los cambios constantes que demanda la economía mundial en aras del desarrollo, y la innovación se ha convertido en un elemento clave en este proceso. No obstante, tal como lo anticiparon Navarro y Olivari (2016), muchas de estas economías presentan un déficit en la incorporación de conocimiento y tecnología a sus procesos productivos debido a los inhibidores que surgen de la complejidad de sus contextos sociales y económicos, lo que no solo pone en riesgo el cumplimiento de las metas de desarrollo, sino que incrementa el riesgo de que la brecha con los países industrializados se perpetúe y se haga incluso más amplia en el tiempo.

Con datos del Índice Global de Innovación de 2016 y apoyo de la metodología de análisis envolvente de datos, en este trabajo se evalúa la eficiencia de las 19 economías que conforman la región de América Latina y el Caribe en materia de creación de entornos favorables para la innovación. La intención es identificar los principales retos de estos países en la gestión y el aprovechamiento de lo que se suele entender como insumos dispuestos para ello, más allá del nivel de ingresos de estas economías. De hecho, los países con mayor nivel de ingreso en América Latina y el Caribe no fueron punteros a nivel de gestión de la eficiencia, por lo que se confirma una modesta correlación entre estas variables.

Además, los países que se encuentran en las mejores posiciones del índice general de eficiencia (Chile, Costa Rica y México) o en las fronteras de la eficiencia regional (Brasil, Chile, Colombia, Costa Rica y Uruguay) no siempre pueden mantener dicha posición si lo que se evalúa es el uso de los insumos para alcanzar determinados productos de innovación, sobre todo por la presencia de rendimientos decrecientes a escala para estas economías, lo que les impide alcanzar mejores

22 Cabe señalar que la información del cuadro 5 no resulta del análisis de eficiencia desarrollado en este trabajo, sino del análisis de los datos de la fuente citada a través de estadística descriptiva. 
resultados a medida que sus insumos son mayores. Esto se hace evidente cuando se contrastan las posiciones punteras de Chile y Colombia en el índice general para América Latina y el Caribe con las ubicaciones por debajo de media tabla en la razón de eficiencia.

El mejor desempeño en cuanto a eficiencia técnica global y eficiencia técnica pura se presentó en el pilar de infraestructura. El peor comportamiento medio en eficiencia técnica global se registró en el pilar de capital humano e investigación. En cuanto a eficiencia técnica pura, el peor caso fue en el pilar institucional, y en eficiencia técnica de escala fue la sofisticación del mercado, que delata dificultades en el desarrollo de los mercados financieros y de competencia comercial. Por otra parte, si bien la mayoría de las relaciones individuales entre insumos y productos son positivas en América Latina y el Caribe (aunque menos que las que ocurren en otras regiones, como América del Norte o Europa), en las relaciones no positivas suelen estar involucrados indicadores como educación terciaria, I+D, crédito e inversión. Esto es preocupante porque estas variables deberían constituir la base de la creación de un entorno que favorezca el desarrollo innovador. Tampoco los trabajadores del conocimiento y los vínculos de innovación parecen tener fuerte impacto en los productos de innovación, mientras que las limitaciones de capacidades impiden que la absorción del conocimiento pueda ser convenientemente aprovechada en la obtención de resultados de eficiencia.

Los países de América Latina y el Caribe tienen una arraigada tradición en materia de políticas de desarrollo científico y tecnológico (OCDE/Eurostat, 2005), que ha terminado por incidir en el número de programas de fomento a la innovación puestos en marcha y en el nivel de participación por parte de los distintos actores. No obstante, los lastres que arrastra la región, marcada por debilidades institucionales (incluidos los aspectos regulatorios), inestabilidad económica, actividades informales y problemas de corrupción (Schwab, 2016), anticipan que será una tarea de largo aliento poder posicionar a los países que la conforman en los primeros lugares del Índice Global de Innovación a través de una gestión eficiente de los insumos que, además, se refleje en un mayor bienestar social y crecimiento económico.

Aunque la mayoría de las políticas y acciones llevadas a cabo por los países de la región para el fomento de los entornos innovadores son congruentes con sus capacidades y ventajas relativas en un contexto heterogéneo (Bárcena, 2008), quedan algunos desafíos pendientes. Uno de ellos radica en fortalecer los insumos que históricamente han mostrado un desempeño pobre y, sobre todo, aprovechar de manera estratégica las relaciones que probadamente han resultado más sólidas entre insumos y productos, de forma tal que se maximice su impacto, primero en la productividad y después en el desarrollo.

Cabe hacer notar que el presente trabajo corresponde a un análisis parcial de la eficiencia de los países de América Latina y el Caribe, ya que - por limitaciones técnicas - en él solo se da cuenta de las interrelaciones más representativas del fenómeno con base en la literatura. Además, las limitaciones de los resultados se hacen evidentes al no haberse incluido un seguimiento a las inversiones públicas y privadas en innovación y desarrollo, su costo de oportunidad o el grado de cumplimiento de las metas concretas de cada país en la materia. Es decir que se ha evaluado la relación entre insumos y productos de innovación, pero no los procesos y mecanismos que los vinculan. Tampoco se han tomado en cuenta las posibles prioridades nacionales con base en la heterogeneidad de la región.

En lo que concierne a las líneas de investigación futura, resulta relevante identificar los criterios que permitan incorporar variables asociadas al impacto en la productividad y el desarrollo humano. Hay que tener en cuenta, también, que considerar el papel que juega el sector público, el grado de logro de las metas locales y su congruencia estratégica podría hacer que las conclusiones tuvieran un mayor alcance. 


\section{Bibliografía}

Álvarez, A. (coord.) (2001), La medición de la eficiencia y la productividad, Madrid, Ediciones Pirámide.

Atilano, Á., J. Mercado y H. Casanova (2015), Indicadores de innovación tecnológica de los países de América Latina y el Caribe - 2015, Caracas, Banco de Desarrollo de América Latina (CAF).

Banco Mundial (2010), Innovation Policy: A Guide for Developing Countries, Washington, D.C.

Banker, R. y otros (1990), "An introduction to data envelopment analysis with some of their models and its uses", Research in Governmental and Nonprofit Accounting, vol. 5, Emerald Group Publishing.

Bárcena, A. (ed.) (2008), "Innovación para el desarrollo: reflexiones desde América Latina y el Caribe", presentación realizada en el IV Encuentro Empresarial Iberoamericano, San Salvador, 29 de octubre.

Bogliacino, F. y otros (2009), "Innovation in developing countries: the evidence from Innovation Surveys", Milán, Universidad de Bocconi [en línea] http://citeseerx.ist.psu.edu/viewdoc/download?doi=10.1.1.45 5.3127\&rep=rep $1 \&$ type $=$ pdf.

Broekel, T., N. Rogge y T. Brenner (2018), "The innovation efficiency of German regions - a shared-input DEA approach", Review of Regional Research, vol. 38, № 1, Springer.

Cañibano, C. (2005), "El capital humano: factor de innovación, competitividad y crecimiento" [en línea] http:// www.navarra.es/nr/rdonlyres/d696efd2-6aaa-4ef1-b414-e3a27109ea67/79785/14carolinacaibano.pdf.

Carro, R. y D. González (2015), Administración de las operaciones: actividades para el aprendizaje, Mar del Plata, Universidad Nacional de Mar del Plata.

Coelli, T. y otros (2003), Una introducción a las medidas de eficiencia para reguladores de servicios públicos y de transporte, Bogotá, Banco Mundial/Alfaomega Colombiana S.A.

Comisión Europea (1995), Libro verde de la innovación, Bruselas.

Etzkowitz, H. y L. Leydesdorff (2000), "The dynamics of innovation: from national systems and 'mode 2' to a triple helix of university-industry-government relations", Research Policy, vol. 29, № 2, Amsterdam, Elsevier.

Fundación COTEC (2001), Innovación tecnológica: ideas básicas, Madrid.

Guimón, J. (2004), "Relevance and impact of intangibles disclosure in capital markets", Lecturas sobre intangibles y capital intelectual, L. Cañibano y P. Sánchez (eds.), Madrid, Asociación Española de Contabilidad y Administración de Empresas.

Hashimoto, A. y M. Kodama (1997), "Has livability of Japan gotten better for 1956-1990?: a DEA approach", Social Indicators Research, vol. 40, N³, Springer.

Kline, S. y N. Rosenberg (1986), "An overview of innovation", The Positive Sum Strategy: Harnessing Technology for Economic Growth, R. Landau y N. Rosenberg (eds.), Washington, D.C., The National Academies Press.

Lo, F., Ch. Chien y J. T. Lin (2001), "A DEA study to evaluate the relative efficiency and investigate the district reorganization of the Taiwan power company", IEEE Transactions on Power Systems, vol. 16, № 1.

Martínez, F., J. C. de Miguel y P. Murias (2005), "El análisis envolvente de datos en la construcción de indicadores sintéticos: una aplicación a las provincias españolas", Estudios de Economía Aplicada, vol. 23, № 3, Valladolid, Asociación Internacional de Economía Aplicada (ASEPELT).

Martínez, F. y P. Murias (2011), "Sistemas de pensiones y bienestar económico de la población mayor: un indicador sintético para los países de la OCDE", Revista Galega de Economía, vol. 20, Santiago de Compostela, Universidad de Santiago de Compostela.

Navarro, J. C. y J. Olivari (eds.) (2016), La política de innovación en América Latina y el Caribe: nuevos caminos, Washington, D.C., Banco Interamericano de Desarrollo (BID).

Navarro, J. C. y Z. Torres (2006), "Análisis de la eficiencia técnica global mediante la metodología DEA: evidencia empírica en la industria eléctrica mexicana en su fase de distribución, 1990-2003", Revista Nicolaita de Estudios Económicos, vol. 1, №1, Michoacán, Instituto de Investigaciones Económicas y Empresariales.

OCDE (Organización de Cooperación y Desarrollo Económicos) (2012), Innovation for Development: A Discussion of the Issues and an Overview of Work of the OECD Directorate for Science, Technology and Industry, París, OECD Publishing.

OCDE/Eurostat (Organización de Cooperación y Desarrollo Económicos/Oficina de Estadística de las Comunidades Europeas) (2005), Manual de Oslo: guía para la recogida e interpretación de datos sobre innovación, tercera edición, París, OECD Publishing.

Quindós, M., F. Rubiera y M. Vicente (2003), Análisis envolvente de datos: una aplicación al sector de los servicios avanzados a las empresas del Principado de Asturias, Rect@, Actas 11, № 1. 
Restrepo, M. y J. Villegas (2007), "Análisis envolvente de datos: introducción y herramienta pública para su utilización", Universidad de Antioquia [en línea] https://juangvillegas.files.wordpress.com/2013/08/ restrepo-villegas-dea.pdf.

Rothwell, R. (1994), "Towards the fifth-generation innovation process", International Marketing Review, vol. 11, $N^{\circ}$ 1, Bingley, Emerald Publishing.

Saren, M. (1984), "A classification and review of models of the intra-firm innovation process", R\&D Management, vol. 14, No 1, Wiley.

Schwab, K. (2016), The Global Competitiveness Report 2016-2017, Ginebra, Foro Económico Mundial.

Universidad de Cornell/INSEAD/OMPI (Organización Mundial de la Propiedad Intelectual) (2016), The Global Innovation Index 2016: Winning with Global Innovation, Ginebra. 